${ }^{9}$ K. W. Schwarz and R. W. Stark, Phys. Rev. Letters $\underline{22}, 1278$ (1969).

${ }^{10} \mathrm{~F}$. Reif and L. Meyer, Phys. Rev. 119, $1164(1960)$.

${ }^{11}$ B. A. Brody, Ph. D. thesis (University of Michigan, 1970) (unpublished).

${ }^{12} \mathrm{~K}$. W. Schwarz (unpublished).

${ }^{13}$ R. M. Bowley, J. Phys. C $\underline{4}, 853$ (1971); 4, 1645 (1971).

${ }^{14}$ I. Iguchi, J. Low Temp. Phys. 4, 637 (1971).

${ }^{15}$ I. M. Khalatnikov and V. Zharkov, Zh. Eksperim. i Teor. Fiz. 32, 1108 (1957) [Sov. Phys. JETP 5, 905 (1957)].

${ }^{16}$ See, for example, I. M. Khalatnikov, Introduction to the Theory of Superfluidity (Benjamin, New York, 1965), Chap. VII.

${ }^{17}$ For example, J. M. Ziman, Electrons and Phonons (Oxford U.P., Oxford, England, 1960), p. 278.

${ }^{18}$ B. D. Josephson and J. Lekner, Phys. Rev. Letters
23, 111 (1969).

${ }^{19}$ J. Ruvalds, Phys. Rev. Letters 27, 1769 (1971); G.

Baym and D. Pines (unpublished).

${ }^{20}$ A. D. B. Woods (unpublished).

${ }^{21}$ D. G. Henshaw and A. D. B. Woods, Phys. Rev. 121, 1266 (1961).

${ }^{22}$ We are grateful to Mr. D. M. Saul for assistance with this calculation.

${ }^{23} \mathrm{M}$. Kuchnir, Ph. D. thesis (University of Illinois, 1966) (unpublished).

${ }^{24}$ We take the Stokes-law mobility calculation from Ref. 3 but we normalize the data with the experimental points of Ref. 23 rather than with the measurements of Ref. 10 .

${ }^{25}$ W. H. Keesom, Helium (Elsevier, Amsterdam, 1942), p. 237.

${ }^{26}$ I. S. Gradshteyn and I. M. Ryzhik, Table of Integrals, Series, and Products (Academic, New York, 1965), p. 316.

PHYSICAL REVIEW A

\title{
Self-Defocusing of Light by Adiabatic Following in Rubidium Vapor
}

\author{
D. Grischkowsky and J. A. Armstrong* \\ IBM Thomas J. Watson Research Center, Yorktown Heights, New York 10598
}

(Received 3 March 1972)

\begin{abstract}
The narrow-line output of a dye laser on the low-frequency side of the ${ }^{2} P_{1 / 2}$ resonance line $(7948 \AA)$ of rubidium was self-defocused by passage through dilute rubidium vapor. The defocusing was caused by the electronic nonlinearity associated with "adiabatic following" of the laser field by the pseudomoment of the resonant atoms. By using the corresponding nonlinear dielectric constant, the wave equation was solved numerically and gave excellent quantitative agreement with experiment.
\end{abstract}

\section{INTRODUCTION}

In the experiments on thermal self-defocusing of light, the intensity dependence of the dielectric constant $\epsilon$ was due to absorption and subsequent heating. ${ }^{1-3}$ The self-defocusing reported here is due to the resonant nonlinearity associated with adiabatic following, and the observations provide a precise test of the adiabatic following model.

Previously, Grischkowsky ${ }^{4}$ observed the selffocusing of light by potassium vapor when the frequency $\nu$ of the light was greater than the frequency $\nu_{0}$ of the ${ }^{2} P_{3 / 2}$ resonance line $(7665 \AA)$ of potassium; however, when $\nu<\nu_{0}$, no self-focusing was observed. Instead the observations were consistent with self-defocusing. These results could be explained either by the adiabatic following model, which was introduced in Ref. 4 , or by the steadystate model of Javan and Kelley. ${ }^{5}$ Both models give constitutive relations which are local and known analytically for all intensities. Also, the resonant electronic nonlinearity of each model causes self-focusing when $\nu_{0}<\nu$ and self-defocusing when $\nu<\nu_{0}$. Recently, Akhmanov et al. ${ }^{6} \mathrm{ob}-$ served self-focusing and self-defocusing in potas- sium vapor. In a limited region they were able to show experimentally that the nonlinear susceptibility was proportional to $\left(\nu-\nu_{0}\right)^{-3}$. This result is obtained from either adiabatic following or the steady-state model.

The term adiabatic following describes the situation in which the pseudomoment $\vec{p}$ of the near resonant transition follows (remains parallel to) the effective field $\vec{B}_{e}$ of the laser pulse. ${ }^{4,7,8}$ Adiabatic following occurs when two conditions are satisfied. First, in the rotating coordinate frame, the direction of $\overrightarrow{\mathcal{S}}_{e}$ must change slowly compared to the precession frequency $\Delta$ of $\vec{p}$ about $\overrightarrow{\mathscr{E}}_{e}\left(\overrightarrow{\mathcal{B}}_{e}\right.$ must change adiabatically $)^{9}$; second, the pulse width must be short compared to $T_{1}$ and $T_{2}$ of the atomic system. The response time of $\vec{p}$ and of the corresponding resonant electronic nonlinearity to changes in $\overrightarrow{\mathcal{S}}_{e}$ is of the order of $\Delta^{-1}$ (for the work reported here the response time was less than 100 psec). In contrast to adiabatic following, the steady-state model is insensitive to how the pulse is applied and requires the pulse duration be long compared to both $T_{1}$ and $T_{2}$. The response time for the steady-state model is approximately $T_{1}$.

We will now derive the condition for adiabatic 
application of the laser field. The experiments of this paper were done with the rubidium vapor in a magnetic field in order to remove all degeneracies. The incident light was circularly polarized and propagated along the field. The electric field of the input laser light can be represented by

$$
\overrightarrow{\mathrm{E}}=E\left[\overrightarrow{\mathrm{e}}_{1} \cos (k z-\omega t-\phi)-\overrightarrow{\mathrm{e}}_{2} \sin (k z-\omega t-\phi)\right],
$$

where $\overrightarrow{\mathrm{e}}_{1}$ and $\overrightarrow{\mathrm{e}}_{2}$ are orthogonal unit vectors. In the coordinate frame $\Sigma^{1}$, rotating about the $z$ axis with angular frequency $\omega=2 \pi \nu, \overrightarrow{\mathrm{E}}$ is given by

$$
\overrightarrow{\mathrm{E}}=E\left(\overrightarrow{\mathrm{e}}_{1}^{1} \cos \phi+\overrightarrow{\mathrm{e}}_{2}^{1} \sin \phi\right) \text {. }
$$

In $\sum^{1}$, the effective field is

$$
\overrightarrow{\mathcal{S}}_{e}=\overrightarrow{\mathrm{E}}+\overrightarrow{\mathrm{e}}_{3}^{1} h\left(\nu-\nu_{0}\right) /\left(p_{12} \sqrt{2}\right),
$$

where $p_{12}$ is the absolute value of the matrix element of the electric dipole moment of the $\sigma$ transition of frequency $\nu_{0} \cdot \overrightarrow{\mathcal{S}}_{e}$ can be rewritten as $\overrightarrow{\mathcal{E}}_{e}=\left|\overrightarrow{\mathscr{E}}_{e}\right|\left[\overrightarrow{\mathrm{e}}_{1}^{1}(\sin \theta)(\cos \phi)+\overrightarrow{\mathrm{e}}_{2}^{1}(\sin \theta)(\sin \phi)+\overrightarrow{\mathrm{e}}_{3}^{1} \cos \theta\right]$, where $\sin \theta=E /\left|\overrightarrow{\mathfrak{B}}_{e}\right|$ and

$$
\cos \theta=h\left(\nu-\nu_{0}\right) /\left(\left|\overrightarrow{\mathcal{E}}_{e}\right| p_{12} \sqrt{2}\right) \text {. }
$$

The precession frequency of $\overrightarrow{\mathrm{p}}$ about $\overrightarrow{\mathcal{S}}_{e}$ is given by

$$
\Delta=\left[\left(\nu-\nu_{0}\right)^{2}+2 p_{12}^{2} E^{2} / h^{2}\right]^{1 / 2} .
$$

The angular rate of change $d \eta / d t$ of the direction of $\overrightarrow{\mathcal{E}}_{e}$ is

$$
\frac{d \eta}{d t}=\left|\overrightarrow{\mathcal{E}}_{e} \times \frac{d \overrightarrow{\mathcal{E}}_{e}}{d t}\right| /\left|\overrightarrow{\mathcal{E}}_{e}\right|^{2},
$$

which is the same as

$$
\frac{d \eta}{d t}=\left[\left(\frac{d \theta}{d t}\right)^{2}+\left(\frac{d \phi}{d t}\right)^{2} \sin ^{2} \theta\right]^{1 / 2} .
$$

$\overrightarrow{\mathcal{S}}_{e}$ changes adiabatically when $d \eta / d t \ll 2 \pi \Delta$. The condition $d \theta / d t \ll 2 \pi \Delta$ is the one usually stated for adiabatic fast-passage experiments in spin resonance where the driving field has very high coherence and $d \phi / d t$ can be neglected. For optical experiments, $d \phi / d t$ can also be neglected if the linewidth is determined by the pulse duration. ${ }^{8}$ However, for many optical experiments the linewidth of the incident light is not determined by the pulse duration and $d \theta / d t<d \phi / d t$. This is probably the situation for the experiment of this paper and was certainly the case for the work of Grischkow$\mathrm{sky}^{4}$ and of Akhmanov et al. ${ }^{6}$ Now $(d \eta / d t) / 2 \pi$ is of the order of $\delta \nu$, the linewidth of the incident light, and to a good approximation $d \eta / d t \ll 2 \pi \Delta$, if $\delta \nu \ll \Delta$. As $\left|\nu-\nu_{0}\right|<\Delta$, a more restrictive requirement but one easier to apply is $\delta \nu \ll\left|\nu-\nu_{0}\right|{ }^{10}$

The analytic expression for $\epsilon$ resulting from adiabatic following is given by

$$
\epsilon=1-\frac{4 \pi N_{e} p_{12}^{2}}{h\left(\nu-\nu_{0}\right)\left(1+2 E^{2} / E_{s}^{2}\right)^{1 / 2}} .
$$

$N_{e}$ is the effective number density of atoms and $E_{s}=\left|h\left(\nu-\nu_{0}\right) / p_{12}\right| .{ }^{11}$ When $E^{2} \ll E_{s}^{2}, \epsilon \approx \epsilon_{0}+\epsilon_{2} E^{2}$, with

$$
\text { and } \begin{aligned}
\epsilon_{0} & =1-4 \pi N_{e} p_{12}^{2} /\left[h\left(\nu-\nu_{0}\right)\right] \\
\epsilon_{2} & =4 \pi N_{e} p_{12}^{4} /\left[h\left(\nu-\nu_{0}\right)\right]^{3} .
\end{aligned}
$$

\section{EXPERIMENT}

The experimental apparatus is diagramed in Fig. 1. The dye laser (DTTC in methanol) was longitudinally pumped by a $Q$-switched ruby laser and produced a $2-\mathrm{mm}$-diam beam with a peak power of several kilowatts, a pulse width of $10 \mathrm{nsec}$, and a full divergence of less than $1 \mathrm{mrad}$. The dyelaser output was frequently a single longitudinal mode with a linewidth $\delta \nu / c$ less than $0.005 \mathrm{~cm}^{-1}$, as shown by very little beating in the detector output and by Fabry-Perot interferograms (see Fig. 2 ). More often the output was multimode and consisted of one dominant mode separated by 0.03 $\mathrm{cm}^{-1}$ from one or two adjacent longitudinal modes oscillating weakly. The narrow-line output of the dye laser was obtained by including in the cavity a combination of two high finesse $(\mathcal{F} \approx 60)$ Fabry Perot interferometers with slightly incommensu-

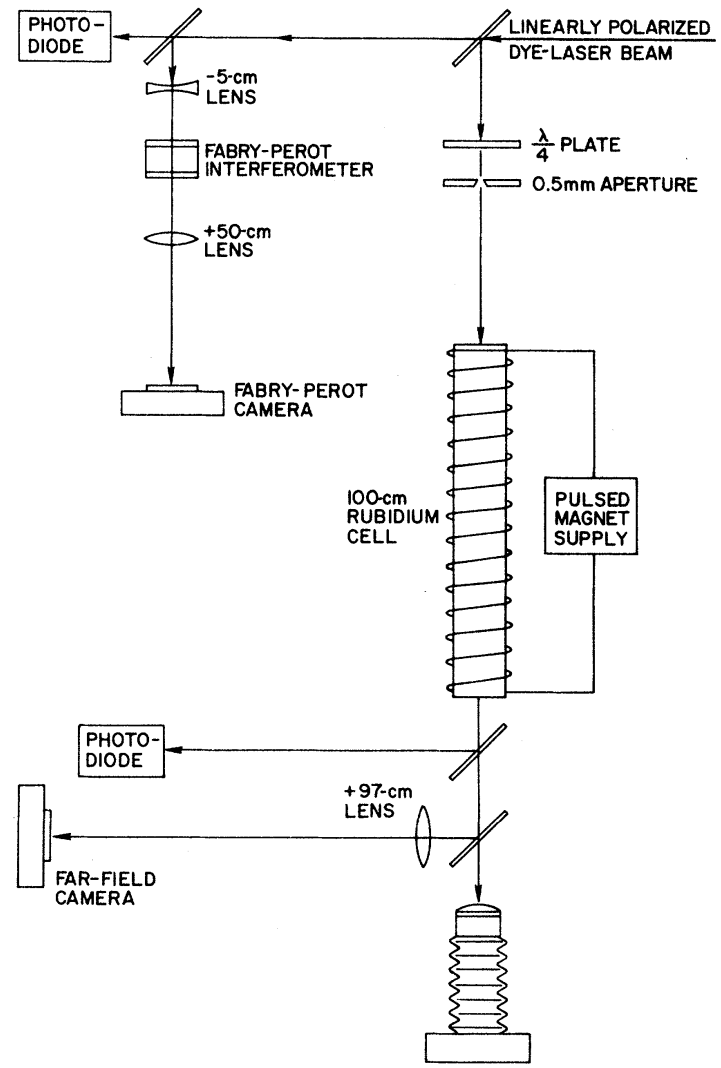

FIG. 1. Schematic diagram of the experiment. 


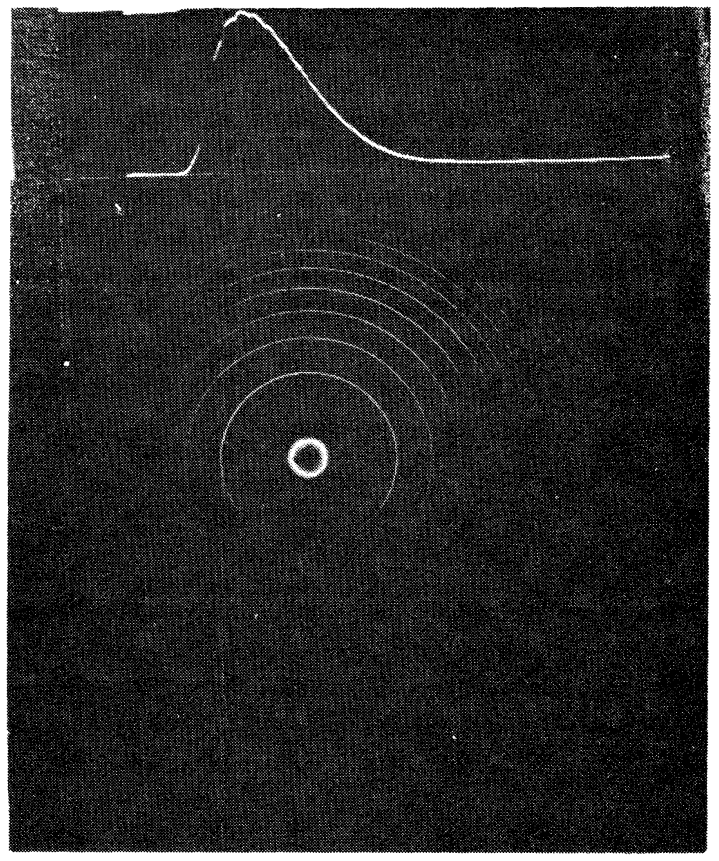

FIG. 2. Single-mode $\sigma^{-}$dye-laser pulse $(10-n s e c$ pulse width) of Fig. 3 observed with an ITT biplanar photodiode and a Tektronix 519 oscilloscope. A FabryPerot interferogram of the same pulse. The interferometer had a finesse of $\approx 100$ and a spectral range of 0.33 $\mathrm{cm}^{-1}$. The measured linewidth was less than $0.005 \mathrm{~cm}^{-1}$.

rate spectral ranges of the order of $13 \mathrm{~cm}^{-1}$. The dye laser oscillated where the transmission maxima of the two interferometers overlapped, and the frequency of this intersection could be tuned continuously by tilting the interferometers with respect to the cavity axis.

A Fabry-Perot interferogram and an oscillogram (Fig. 2) were taken of each pulse of the dye laser before the beam was circularly polarized (the helicity could be changed from $\sigma^{+}$to $\sigma^{-}$by rotating a quarter-wave plate $90^{\circ}$ ) and encountered a centered $0.5-\mathrm{mm}$-diam aperture. The aperture was $50 \mathrm{~cm}$ from the entrance window of the $100-\mathrm{cm}$ rubidium-vapor cell, so the transverse intensity distribution of the beam entering the cell was the Fraunhofer diffraction pattern of the aperture. We note that when beam-defining apertures are used outside the laser, as in this case, it is very important that the aperture be far enough from the cell input for the beam to be described by Fraunhofer rather than Fresnel diffraction. This is because it is extremely inconvenient to describe Fresnel diffraction from a circular aperture analytically and hence very difficult to compare theory and experiment. After passage through the cell near-field and far-field, photographs (Fig. 3) were taken of the beam, and the output pulse was moni- tored. For one series of experiments, Fabry Perot interferograms were taken of the emergent beam.

The 8-kG field caused a $1.0-\mathrm{cm}^{-1}$ Zeeman splitting, removed all degeneracies, and put the hyperfine interaction in the Paschen-Bach limit. An excess of rubidium metal was doubly distilled into the Pyrex glass cell, which was then sealed under vacuum. Increasing the cell temperature increased $N$ and consequently increased $\left|\epsilon_{2}\right|$. The density vs-exposure curve ( $H-D$ curve) of the Kodak highspeed infrared film 2481 was obtained with a single pulse of the dye laser by placing the film $50 \mathrm{~cm}$ from the aperture and using the observed Fraunhofer rings as the exposure calibration.

\section{RESULT}

Since the experiments were done with the $R b$ vapor in an 8-kG magnetic field, we were able to study the interaction of $\sigma^{+}$and $\sigma^{-}$circularly polarized light separately, and hence verify the strongly resonant nature of the nonlinearity. For the results shown in Figs. 3 and $4, \epsilon_{2}=-2.5 \times 10^{-7}$ esu for $\sigma^{-}$light, which was 33.4 times larger than $\epsilon_{2}$ for $\sigma^{+}$light. For $\sigma^{-}$light, $\nu_{0}-\nu=0.45 \mathrm{~cm}^{-1}$, and for $\sigma^{+}$light, $\nu_{0}-\nu=1.45 \mathrm{~cm}^{-1} ; \epsilon_{2}\left(\sigma^{-}\right) / \epsilon_{2}\left(\sigma^{+}\right)$ $=(1.45 / 0.45)^{3}=33.4$. For comparison, $\epsilon_{2}=+4.2$ $\times 10^{-11}$ esu for $\mathrm{CS}_{2}$, one of the most nonlinear Kerr liquids.

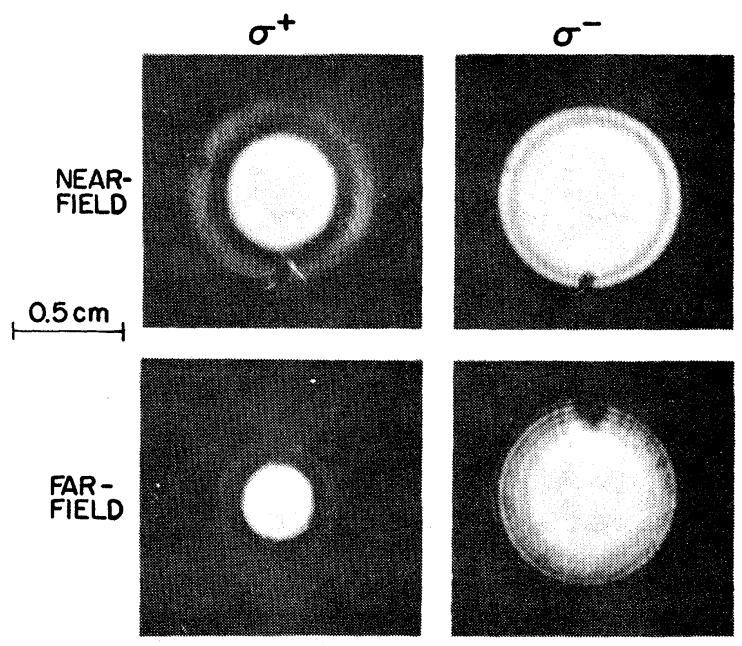

FIG. 3. Near-field and far-field photographs of the 10nsec single-mode dye-laser pulses after passage through the $100-\mathrm{cm}$ rubidium-vapor cell at a temperature of $124^{\circ} \mathrm{C}$ and in a magnetic field of $8 \mathrm{kG}$ vs the direction of rotation $\left(\sigma^{*}\right.$ or $\left.\sigma^{-}\right)$of the circularly polarized input light. The frequency of the $\sigma^{*}$ light was $1.45 \mathrm{~cm}^{-1}$ below the frequency of the $\sigma^{+}$component of the Zeeman-split ${ }^{2} P_{1 / 2}$ line $(7948 \AA)$ of rubidium, while the $\sigma^{-}$light was $0.45 \mathrm{~cm}^{-1}$ below the $\sigma^{-}$component. For the $\sigma^{+}$pulse the peak power was $185 \mathrm{~W}$, and for the $\sigma^{-}$pulse (shown in Fig. 2) the peak power was $235 \mathrm{~W}$. 
The observed defocusing for the two polarizations is shown in Fig. 3. The pictures labeled "near-field" are the intensity distribution photographed at the end window of the $100-\mathrm{cm} \mathrm{Rb}$ cell. The far-field pictures were obtained in the focal plane of a $97-\mathrm{cm}$ focal length lens. The two polarizations show qualitatively different behavior. The distribution of $\sigma^{+}$light deviates only slightly from a Fraunhofer diffraction pattern, corresponding to the input pulse having had an accurate Fraunhofer pattern. The distribution of the $\sigma^{-}$ light (which also had a Fraunhofer distribution at the cell input) has been strongly modified. In the near-field, the peak has been flattened and broadened, and two rings (the outer ring is easily seen on the negative) have appeared where there would be one in the Fraunhofer pattern. Also, in the far-field the central peak has broadened and rings have appeared.

The near-field calculation was similar to Kelley's, ${ }^{12}$ but with modifications appropriate to our problem. The input beam was accurately a Fraunhofer diffraction pattern and was therefore represented for the calculation by the function

$$
E_{0}\left(\frac{2 J_{1}(k a r / D)}{k a r / D}\right) e^{i k r^{2} / 2 D} .
$$

Here $r$ is the radial coordinate in the input plane, $a$ is the radius of the diffracting aperture, and $D$ is the distance from the aperture to the input plane. The axial and radial distances are normalized in the usual way, but the field is normalized to its saturation value. In normalized form the selfdefocusing equation is therefore

$$
\begin{aligned}
\frac{i \partial \bar{E}}{\partial \bar{z}}+\frac{1}{r} \frac{\partial \bar{E}}{\partial \bar{r}}+\frac{\partial^{2} \bar{E}}{\partial \bar{r}^{2}}+\frac{k^{2} a^{2} 4 \pi N_{e} p_{12}^{2}}{\epsilon_{0} h\left(\nu-\nu_{0}\right)} & \\
& \times\left[1-\left(1+2|\bar{E}|^{2}\right)^{-1 / 2}\right] \bar{E}=0 .
\end{aligned}
$$

Here $\bar{r}=r / a, k=2 \pi v\left(\epsilon_{0}\right)^{1 / 2} / c, \bar{z}=z / 2 k a^{2}$, and $\bar{E}=E / E_{s}$. The computer program was tested by using the Fraunhofer pattern as input to the "empty" $100-\mathrm{cm}$-long cell. The computed output pattern was almost exactly the Fraunhofer pattern appropriate to a distance $150 \mathrm{~cm}$ from the beam-defining aperture. In matching theory to the experimental results in Fig. 4, it was necessary to take into account the time variation of the laser pulse. This was not critical in dealing with the $\sigma^{+}$pattern, since it is so little changed from the linear case, but for the $\sigma^{-}$light it was important. The $\sigma^{+}$and $\sigma^{-}$pulses were represented by histograms shown in Fig. 4. The defocusing was calculated for each power and the intensities then combined using weights determined simply from the relative area under each power.

The measured values of $\nu_{0}-\nu$ and peak power were used in the computer computation; the value of the number density $N$ at the measured average cell temperature of $124{ }^{\circ} \mathrm{C}$ was obtained from published data and extrapolations of the vapor-pressure curve of $\mathrm{Rb}^{13-15}$; the vapor-pressure data have a scatter from $N=1.3 \times 10^{13} / \mathrm{cm}^{3}$ to $2.5 \times 10^{13} /$ $\mathrm{cm}^{3}$ at $124{ }^{\circ} \mathrm{C}$. The good agreement (shown in Fig. 4) was obtained with $N=2 \times 10^{13}$, which was well within the estimated error of the vapor-pressure curve. The theoretical result was quite sensitive to the input parameters $E_{0}^{2}, N$, and $\left(\nu_{0}-\nu\right)^{-3}$. A change by a factor 2 for any one of them caused large changes in the near-field result, and theory and experiment no longer agreed. The same value of $N$ was used for both the $\sigma^{+}$and $\sigma^{-}$calculations, since the temperatures were the same. The powers were somewhat different, but the important difference was in the distance from resonance. The agreement is very satisfactory in both cases and indicates that the adiabatic following model for the nonlinear dielectric constant is substantially correct. In this regard we note that $2 E^{2} / E_{s}^{2}=1.2$ in the center of the input beam for the $\sigma^{-}$light, and that therefore it would be incorrect to describe the nonlinear index in terms of the first-order expansion $\epsilon_{2} E^{2}$.

For all our experiments the strength of the selfdefocusing increased smoothly with cell tempera-

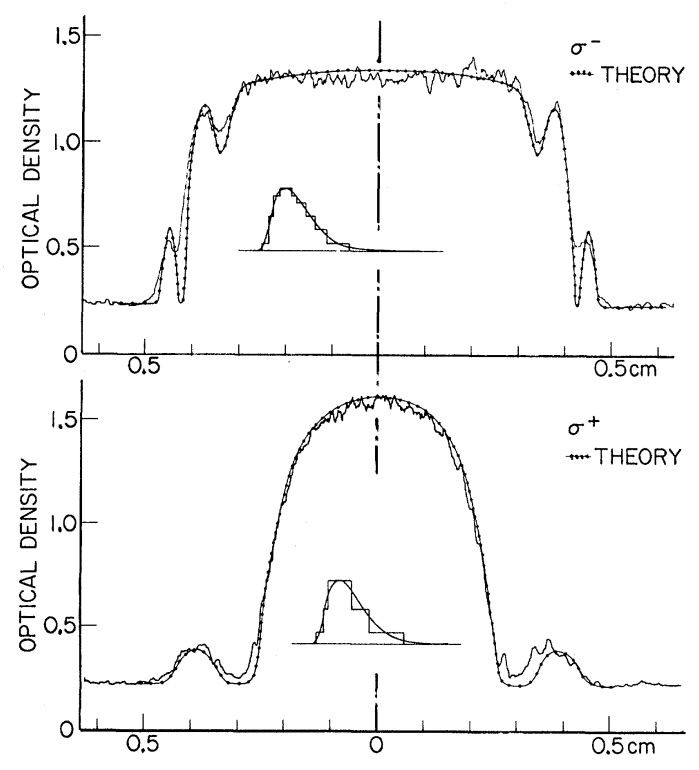

FIG. 4. Comparison of the densitometer traces of the negatives used for the near-field photographs in Fig. 3 with the results of computer integration of the nonlinear diffraction equation for the $\sigma^{+}$and $\sigma^{-}$pulses. The solutions were summed over the pulses as shown by the histograms and were adjusted for the measured densityvs-exposure curve of the film. The parameters used in the calculation had their experimentally determined values. 
ture and input-beam intensity. Experiments without the aperture and with beams with irregular transverse intensity distributions showed that the self-defocusing tends to smooth out the transverse intensity profile of the propagating beams. Selfdefocusing always increased the beam divergence in our results. There was no evidence of absorption of light by the vapor, and at $124{ }^{\circ} \mathrm{C}$ for $\sigma^{-}$light an upper limit for the absorption coefficient $\alpha$ is given by the low-level steady-state result $\alpha=0.9$ $\times 10^{-3} \mathrm{~cm}^{-1}$. When multimode dye-laser output was obtained, strong third-order mixing occurred in the rubidium vapor and indicated that the response time of the resonant electronic nonlinearity was much faster than $1 \mathrm{nsec}$, the period of the beat note between adjacent longitudinal modes of the dye laser.

\section{ACKNOWLEDGMENTS}

The authors would like to acknowledge the invaluable assistance of $R$. J. Bennett in all phases of the experiment and the data reduction. Many useful discussions were held with Michael M. T. Loy. We also thank Professor W. Kaiser and Dr. M. Maier for information about their computation of self-focusing.
*Work of this author was partially supported by ONR under Contract No. N00014-70-C-0187.

${ }^{1}$ J. P. Gordon, E. C. C. Leite, R. S. Moore, S. P. S. Porto, and J. R. Whinnery, J. Appl. Phys. 36, 3 (1965).

${ }^{2}$ S. A. Akhmanov, D. P. Krindach, A. V. Migulin, A. P. Sukhorukov, and R. V. Khokhlov, IEEE J. Quantum Electron. QE-4, 568 (1968).

${ }^{3}$ F. W. Dabby, R. W. Boyko, C. V. Shank, and J. R. Whinnery, IEEE J. Quantum Electron. QE-5, 516 (1969).

${ }^{4}$ D. Grischkowsky, Phys. Rev. Letters 24, 866 (1970).

${ }^{5}$ A. Javan and P. L. Kelley, IEEE J. Quantum Electron. QE-2, 470 (1966).

${ }^{6}$ S. A. Akhmanov, A. I. Kovrigin, S. A. Maksimov, and V. E. Ogluzdin, Zh. Eksperim. i Teor. Fiz. Piz'ma v Redaktsiyu 15,186 (1972) [Sov. Phys. JETP Letters 15, $129(1972)]$.

${ }^{7}$ I. D. Abella, N. A. Kurnit, and S. R. Hartmann, Phys. Rev. 141, 391 (1966).

${ }^{8}$ A. V. Nurmikko and S. E. Schwarz, Appl. Phys. Letters 20, 346 (1972).
${ }^{9}$ I. I. Rabi, N. F. Ramsey, J. Schwinger, Rev. Mod. Phys. 26, 167 (1954).

${ }^{10} \mathrm{For}$ temperatures below about $140{ }^{\circ} \mathrm{C}$ the homogeneous linewidth of the ${ }^{2} P_{1 / 2}$ transition is not broadened by resonant collisions (Ref. 13) but is determined by the lifetime $\tau=28 \mathrm{nsec}$ of the excited state. $T_{1}=\tau$, and below $140^{\circ} \mathrm{C}, T_{2}=2 T_{1}$. The dye-laser pulse width was $10 \mathrm{nsec}$, $\delta \nu / c<0.005 \mathrm{~cm}^{-1}$, and $\left|\nu-\nu_{0}\right| / c=0.45 \mathrm{~cm}^{-1}$; consequently, to a good approximation the conditions for adiabatic following were satisfied.

${ }^{11}$ The number density of rubidium atoms in the $M_{J}= \pm \frac{1}{2}$ Zeeman state of the $5^{2} S_{1 / 2}$ level is $N_{e}=\frac{1}{2} N=1 \times 10^{13} / \mathrm{cm}^{3}$ at $124^{\circ} \mathrm{C} ; p_{12}=6.16 \times 10^{-18}$ esu for the matrix element for the $\sigma^{\mp}$ transition between the $5^{2} S_{1 / 2}, M_{J}= \pm \frac{1}{2} \longleftrightarrow 5^{2} P_{1 / 2}$, $M_{J}=\mp \frac{1}{2}$ Zeeman states of the rubidium atom.

${ }^{12}$ P. L. Kelley, Phys. Rev. Letters 15, 1005 (1965).

${ }^{13}$ Ch'en Shang-Yi, Phys. Rev. 58, 884 (1940).

${ }^{14}$ R. E. Honig, RCA Rev. 18, 195 (1957).

${ }^{15} \mathrm{~A}$. N. Nesmeyanov, Vapor Pressure of the Elements (Academic, New York, 1963).

PHYSICAL REVIEW A

VOLUME 6 , NUMBER 4

OCTOBER 1972

\title{
Behavior of Autocorrelation Functions*
}

\author{
Noel Corngold \\ Department of Engineering and Applied Science, \\ California Institute of Technology, Pasadena, California 91109
}

(Received 15 May 1972)

\begin{abstract}
This paper is concerned with the behavior of the autocorrelation and memory functions of statistical mechanics as $t \rightarrow \infty$. A simple calculation shows that for a large class of models leading to power-law behavior, the two functions have opposite sign and common functional behavior in the limit $t \rightarrow \infty$.
\end{abstract}

\section{INTRODUCTION}

The calculation of a typical autocorrelation function (acf) $\Phi(t)$ via an integrodifferential equation

$$
\frac{d}{d t} \Phi(t)=-\int_{0}^{t} d \tau K(t-\tau) \Phi(\tau), \quad \Phi(0)=1
$$

whose kernel is the appropriate "memory function (mf)," is of great utility in modern statistical mechanics. ${ }^{1}$ However, little is known, in general, about the quantities $\Phi(t)$ and $K(t)$. It is the purpose of this paper to point out simple relations which must exist between these functions when the longtime behavior of the acf is of certain nonexponential varieties. These relations are summarized in Eqs. (13). Since there has been considerable in- 


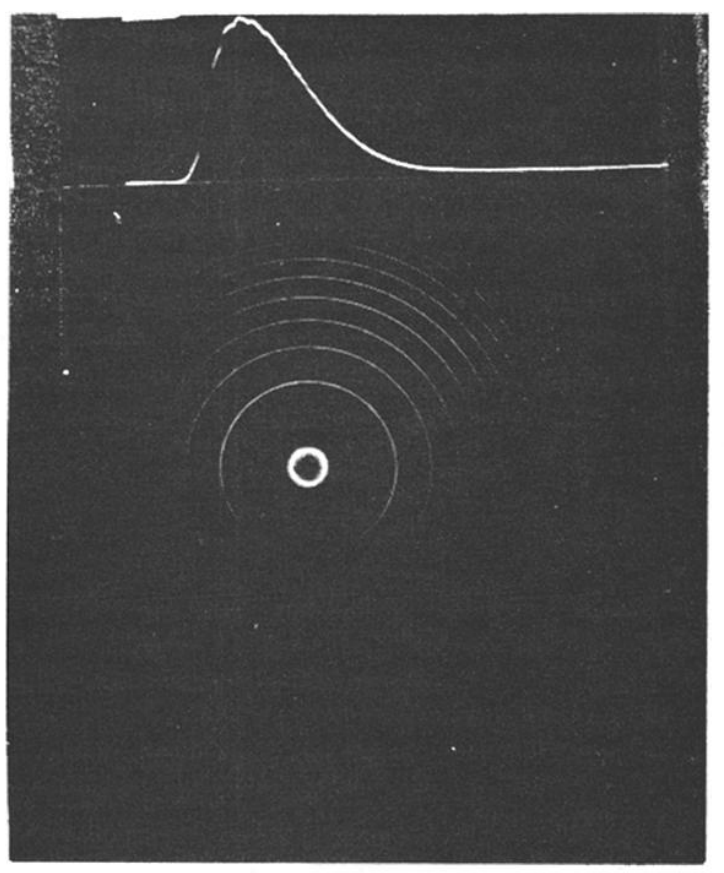

FIG. 2. Single-mode $\sigma^{-}$dye-laser pulse (10-nsec pulse width) of Fig. 3 observed with an ITT biplanar photodiode and a Tektronix 519 oscilloscope. A FabryPerot interferogram of the same pulse. The interferometer had a finesse of $\approx 100$ and a spectral range of 0.33 $\mathrm{cm}^{-1}$. The measured linewidth was less than $0.005 \mathrm{~cm}^{-1}$. 


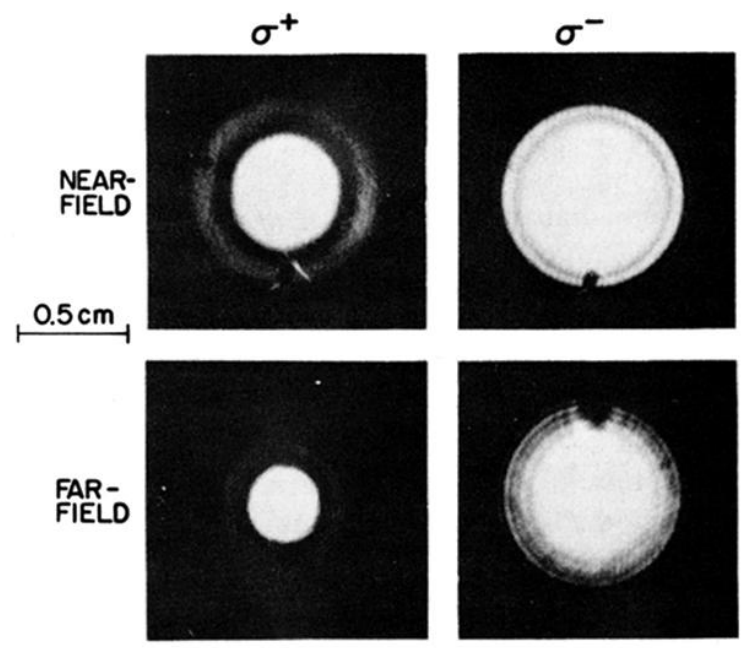

FIG. 3. Near-field and far-field photographs of the 10nsec single-mode dye-laser pulses after passage through the $100-\mathrm{cm}$ rubidium-vapor cell at a temperature of $124^{\circ} \mathrm{C}$ and in a magnetic field of $8 \mathrm{kG}$ vs the direction of rotation $\left(\sigma^{+}\right.$or $\left.\sigma^{-}\right)$of the circularly polarized input light. The frequency of the $\sigma^{+}$light was $1.45 \mathrm{~cm}^{-1}$ below the frequency of the $\sigma^{*}$ component of the Zeeman-split ${ }^{2} P_{1 / 2}$ line $(7948 \AA)$ of rubidium, while the $\sigma^{-}$light was $0.45 \mathrm{~cm}^{-1}$ below the $\sigma^{-}$component. For the $\sigma^{+}$pulse the peak power was $185 \mathrm{~W}$, and for the $\sigma^{-}$pulse (shown in Fig. 2) the peak power was $235 \mathrm{~W}$. 